\title{
In-vitro Evaluation of Ventricular Cannulation for Rotodynamic Cardiac Assist Devices
}

\author{
Timothy N Bachman, MS*, Jay K Bhama, MD ${ }^{\dagger}$, Josiah Verkaik, BS $\ddagger$, Stijn Vandenberghe, \\ PhD§, Robert L Kormos, MD ${ }^{\dagger}$, and James F Antaki, PhD§ \\ *University of Pittsburgh, Pittsburgh, PA, USA \\ tUniversity of Pittsburgh Medical Center, Pittsburgh, PA, USA \\ ‡LaunchPoint Technologies, Inc., CA, USA \\ $\S$ Carnegie Mellon University, Pittsburgh, PA, USA
}

\begin{abstract}
The influence of positioning and geometry of ventricular cannulas for contemporary continuous flow Left Ventricular Assist Devices (LVADs) was evaluated in a non-beating isolated heart preparation with borescopic visualization. Preload and LVAD flow were varied to evaluate degrees of ventricular decompression up to the point of ventricular collapse. The performance of a flanged cannula was compared to a conventional bevel-tipped cannula: quantitatively by the maximal flow attainable, and qualitatively by visualization of fluid tracer particles within the ventricular chamber. Three forms of ventricular suck-down occurred: concentric collapse, gradual entrainment and instantaneous entrainment. In some circumstances, unstable oscillations of the ventricle were observed prior to complete collapse. Under conditions of low preload, the flanged cannula demonstrated less positional sensitivity, provided greater flow, and exhibited fewer areas of stagnation than the beveled cannula. These observations warrant further consideration of a flanged ventricular cannula to mitigate complications encountered with conventional cannulae.
\end{abstract}

\section{Introduction}

The interface between the ventricle and cannula is a non-negligible source of complication for Ventricular Assist Devices (VADs). Current ventricular inflow cannulae have been shown to cause adverse events that result in decreased pump output and increased morbidity and mortality.[1] The use of turbodynamic blood pumps presents a particular challenge to cannulation due to their potential to generate negative inflow pressure causing ventricular suction.[2,3] Current cannula designs have exhibited susceptibility to misalignment and post-operative migration.[4] [5] [6] Incorrect positioning may not become apparent until the chest of a patient has been closed. A misaligned cannula can lead to premature ventricular suction, entrapment of ventricular anatomy, reduction of flow, endocardial trauma, arrhythmia, and/or thrombus formation. The apprehension of suction commonly prompts clinicians to operate the VAD suboptimally. In fact, suction is such a concern that feedbackcontrollers are equipped with suction detectors to respond to this event.[7-9] A more robust

\footnotetext{
${ }^{1}$ Corresponding Author: James F Antaki, PhD, antaki@andrew.cmu.edu, Department of Biomedical Engineering, Carnegie Mellon University, 700 Technology Drive, 4321, Pittsburgh, PA 15219.

Disclosures

At the time of the study, Josiah Verkaik was employed by LaunchPoint technologies, Inc. All remaining authors have no disclosures to report.
} 
cannula design that would alleviate the propensity to mild suction conditions could allow higher VAD flows and more complete decompression of the ventricle.

Prevention of ventricular suction and entrapment has been addressed via simple modifications of pre-existing cannulae by either adding vents or increasing the length of the cannula tip.[10] These remedies may however increase the possibility of thrombus formation due to flow disturbances causing shear activation of platelets and increased exposure time of stagnant blood to exposed myocardium at the apical wound site.

Motivated by the perceived inadequacies of conventional cannula for use with turbodynamic VADs, Antaki et al. introduced a novel "trumpet mouth" cannula that was shown in-vivo to facilitate placement during insertion, provide stenting of the ventricular apex, mitigate regions of flow stasis, and provide greater flows under low volume conditions. [11] Curtis et al. validated the acute findings of Antaki et al. via in-vitro flow visualization of cannulated mock bovine ventricles. There was no region of stagnant flow found with the trumpet mouth. The flow appeared laminar within the steady flow experiments and displayed good washing with pulsatility. [12]

The trumpet cannula prototype was however more difficult to insert, due to the size and rigidity of the flange. This has motivated the development of a second generation cannula having a compliant internal flange that is conformal along the apical portion of the endocardial wall. (See Figure 1) An external flange was added to provide additional retention, thereby obviating a surgical sewing ring. The conduit portion of the cannula was also modified with ribs to prevent kinking and permanent deformation after being cross clamped.

\section{Methods}

Experiments were performed in an non-beating heart preparation described in detail by Bhama et al [13]. Briefly, a flow loop was employed that consisted of a conventional turbodynamic pump (Biomedicus BP80, Medtronic, Inc.), fluid reservoir, flexible tubing, into which the cannulated heart was interposed. Direct visual access to the cannula tip was provided by a borescope introduced through the mitral orifice directed antegrade, towards the lumen of the cannula. (See Figure 2.) The same beveled cannula reported by Bhama et al. (Thoratec Corporation, Pleasanton, CA) was used as a baseline for comparison to the trumpet flanged design. The prototype flanged cannula was fabricated by room-temperature pour molding using $80 \mathrm{~A}$ durometer polyurethane (F-80, BJB Enterprises, Tustin, CA). The prototype cannula was introduced through a stab incision in the ventricular apex, and secured in place with the integrated external flange described previously.[14] No additional suturing or means retention was employed. The loop was primed with distilled water at room temperature and was seeded with neutrally buoyant reflective particles (Amberlite IRA-96, Sigma-Aldrich, St. Louis, MO) to visualize the internal flow characteristics in the region of the cannula tip.

\section{Experimental Design}

A single ovine heart was used for each of two studies in which the two cannulae were consecutively evaluated. Each experiment consisted of two protocols which reproduced two clinically feasible hemodynamic conditions: (1) hypovolemia, often encountered due to bleeding or dehydration and (2) over-pumping, caused when the pump speed (hence, flow) exceeds venous return. The hypovolemic protocol was achieved by restricting venous return using a Hoffman clamp proximal to the heart while maintaining a fixed pump speed. This was completed at three speed settings: 1250, 1500, and 1750 RPM. The over-pumping protocol was performed with a fixed venous resistance while the speed was incrementally 
increased to the point of suction. Three baseline conditions were imposed by adjustment of the Hoffman clamp at an initial speed of 1200 RPM to achieve left atrial pressures (LAP) of 20,15 , and $10 \mathrm{mmHg}$. Both protocols were performed with two cannula orientations: aligned, i.e. collinear with the long axis of the ventricle and misaligned, deliberately deflected posteriorly by an approximate angle of 45 degrees. The above procedure was repeated with a second heart in which the order of the cannula used was reversed for the second study.

\section{Data Acquisition and Analysis}

Continuous data were recorded by personal computer equipped with analog-to-digital board (TB-100, Iotech Inc., Cleveland, OH). In addition to the recording of LAP and flow, the pressure distal to the cannula was recorded immediately distal to the cannula in the first study, and distal to the pump head in the second study. Throughout the studies, the video recordings were made of the interior of the ventricle through the borescope. Real-time observations of patency of the ventricle, cannula, and flow fields were also recorded manually. As ventricular obstruction of flow through the cannula occurred, the mode of cannula occlusion was classified as either: concentric collapse, gradual entrainment, or instantaneous entrainment of the ventricular wall. Hemodynamic data were analyzed offline using Microsoft Excel.

A positional sensitivity index $\left(\mathrm{I}_{\mathrm{PS}}\right)$ was defined as the percentage change in flow due to malpositioning:

$$
I_{P S}=\frac{Q^{\prime}-Q^{\prime \prime}}{Q^{\prime}} * 100 \%
$$

for any given baseline condition. Where $Q^{\prime}$ is the flow recorded at a specific increment of LAP and speed when a cannula is aligned, and $Q^{\prime \prime}$ is the corresponding flow recorded with the cannula misaligned.

\section{Results}

The flow field within the conventionally cannulated ventricle, as observed through the trajectory of particle tracers, was characterized by a laminar central core between the mitral annulus and cannula orifice surrounded by a region of recirculating flow around the cannula tip - as observed previously in-vitro by Curtis et al. [12] Regions of poor washing were identified through the accumulation of tracer particles near the root of the cannula tip. The misaligned cannula also caused particles to accumulate between the cannula outer wall and LV endocardial free wall. (See Figure 3.) Flow fields generated by the flanged tip did not produce such areas of stagnation. Individual particles were occasionally observed to deposit in crevices of trabeculae or at the very edge of the flange, but only for brief periods of time. This was true for both aligned and misaligned flanged cannula.

When pump flow and/or speed were sufficiently increased, one of three modes of ventricular suction was observed. (See Figure 5) The first mode was a concentric collapse, characterized by the walls of the ventricle being drawn symmetrically inward above (proximal to) the cannula orifice. In this condition, volumetric flow was often maintained, albeit limited, due to incomplete coaptation of the ventricular walls. The second mode of suction occurred when only the free wall of the ventricular chamber was entrained by the cannula orifice. This entrainment occurred in one of two ways: instantaneously, in a sudden and nearly total occlusion of the cannula; or gradually, wherein the myocardium was gradually drawn 
towards the cannula, directly related to the flow rate, until the orifice was completely obstructed. The type of suction was dependent upon the cannula geometry and positioning. Suction modes associated with each test condition are listed in Table 1.

During several of the suction events with the flanged cannula in the first study and during nearly all suction events with flanged cannula in the second study, repeated occlusion followed by reopening of the flow path would occur. These oscillations would continue until pump speed was reduced, the ventricle became continuously collapsed, or preload was restored.

Figure 45 illustrates the differences in volumetric flow rate observed between the two cannulae and their respective positioning for both protocols. Alteration of position had only a minor effect on flow at high preloads. For the majority of the experiments, an aligned beveled cannula achieved higher flows than did the aligned flanged cannula. The maximal flows achieved for the beveled and flanged cannulae were $7.1 \mathrm{lpm}$ and $6.6 \mathrm{lpm}$ respectively in the first study, and $6.7 \mathrm{lpm}$ and $6.6 \mathrm{lpm}$ respectively in the second study. As preload decreased, the dependence of flow on positioning of the beveled cannula was greatly accentuated. In contrast, the flanged cannula demonstrated only slight sensitivity to misalignment under similar conditions.

The same trend was observed during the over-pumping protocol. The beveled cannula achieved higher flows before experiencing a suction event than the flanged cannula when both were nominally positioned. However, the maximal achievable flows with the misaligned beveled cannula were well below the corresponding flows achieved with the misaligned flanged design.

The relative (percent) change of flow due to misalignment are provided in Figure 7.. The most dramatic example of positional sensitivity for the hypovolemic protocol was exhibited by the beveled cannula, in which misalignment caused a diminution in flow of nearly $48 \%$ (LAP of $5 \mathrm{mmHg}$ and speed of $1250 \mathrm{RPM}$.) In contrast, the flanged cannula exhibited a drop of less than 5\% at the same LAP and speed. Likewise, the reduction of maximal flows attained during the over-pumping protocol was much more pronounced in the beveled cannula (34.2\% in the first study and $29.5 \%$ in the second study) as compared to the flanged cannula ( $7.3 \%$ and $8.2 \%$, respectively, in the first and second studies.)

The morphology of the flow waveforms was also found indicative of the character of ventricular suck-down. Figure 6 illustrates two types of oscillations observed. The first type was characterized by incomplete interruption of flow, which dampened as the oscillation progressed. The second exhibited intermittent cessation of flow as the orifice of the cannula was completely occluded.

Supplemental movie files (Movies 1-4) illustrating these suction events and oscillations can be found on the Cardiovascular Engineering and Technology website.

\section{Discussion}

The frequency and severity of cannula-related events and complications remain controversial. Published literature is sparse in this regard, however it is commonly understood amongst cardiac surgeons that the cannula is inserted "blindly" into the ventricle. Despite significant experience and intuition, there is currently no definitive means of insuring the intra-ventricular positioning of the cannula inter-operatively. It is further understood that even a well-positioned cannula may become altered due to: closure of the chest, reverse remodeling of the ventricle, and even postural changes. A recent report in the New England Journal of Medicine of an acute ventricular septal defect caused by erosion of 
the LVADs inflow cannula into the septum is described as "an unusual complication." Such a severe case of cannula misalignment resulting in death is indeed rare; but it would imply that less severe cases are less rare. This is supported by a recent study conducted by the Artificial Heart Program at the University of Pittsburgh in which 3-D reconstructed images of the chest revealed remarkably misaligned cannula within the left ventricle, contrary to the surgeon's recollection during surgery [14].

This present study provided visual evidence of various forms of LVAD-induced left ventricular suction in-vitro. It demonstrated that positioning of the cannula can influence the onset of suction, and resulting diminution of flow. It also demonstrated that a conformal cannula tip can decrease this positional sensitivity. On the other hand, the cannula flange was found to affect the characteristic behavior of ventricular suck down. Hemodynamic oscillations observed only with the flared cannula may indicate that the elastic recoil of the internal flange may force open or stent the collapsed ventricle under mild suction conditions. The clinical consequence of this property is not yet understood; however this phenomenon may benefit feedback controllers, by indicating when suction is occurring. There may also be relevance with respect to recovery from suction. One mode of suction may be relieved by simply reducing the pump flow slightly below the suction point, while another may exhibit more hysteresis: requiring a greater reduction of flow. This is a topic of practical importance worthy of further investigation.

Numerous assumptions and simplifications associated with this study limit its direct extrapolation to the clinical setting. Most prominent is the use of a non-viable ovine heart. This experimental preparation was chosen partly for its simplicity, but also for the ability to use an optically clear fluid visualize the ventricular chamber. Our group has in fact implemented a wide range of experimental protocols, each with their advantages and limitations. In-vivo studies of a pediatric ventricular cannula [15] provided the opportunity to study the effects of cardiac contraction: both the biomechanical influence on the cannulamyocardial interface as well as the effect of unsteady, pulsatile flow. However it was not amenable to intraventricular visualization. Isolated beating heart preparations suffer similar limitations inasmuch as large mammalian hearts typically require an oxygen carrying medium, unlike the rodent heart. The use of a transparent blood analog, such as perfluorocarbon may obviate this problem, but would also introduce new complications. To account for the unique geometric anomalies of cardiomyopathy, our group has also conducted flow visualization studies with elastomeric casts of the dilated adult ventricle[12, 16]. These studies however do not well reproduce the phenomenon of ventricular suction. The use of a non-beating heart, although rudimentary, was found to be a reasonable compromise between complexity and accuracy. However it is understood that no solitary experimental preparation can provide all the data desired under clinically-relevant conditions.

A further limitation of the present study is the limited sample size. Although counterintuitive that random chance may be responsible for these observations, it is clear that additional studies are needed to draw statistically significant conclusions. The choice of circulating fluid is another potential source of error. Although a more viscous blood analog, or blood itself, will increase the hydrodynamic resistances, particularly the coarctations of the fluid circuit, this would tend to further accentuate differences between cannulae. The influence of density in these steady-flow studies should be negligible, but would become important in the beating heart because of the predominance of inertial effects. Despite the above limitations, these studies certainly provide pilot data that suggest hemodynamic parameters that should be the focus of future studies. 
The apparent benefits of the flanged cannula, particularly in reducing positional sensitivity to flow obstruction also prompt further investigation. The actual design of this cannula has not yet been fully optimized. Future improvements could be made with respect to the flange diameter, cone angle, and material flexibility to achieve an optimal combination of stenting ability, ease of insertion, and retention. The design could also be improved upon by providing the surgeon with the option of anchoring the cannula with sutures to prevent dislodgement and risk of tamponade. On the other hand, this cannula has previously exhibited excellent retention ability without the need for purse string suture or sewing ring [14] and would therefore be a promising candidate for minimally-invasive or off-bypass surgical insertion.

\section{Conclusion}

The development and use of circulatory assist systems does not typically invite great attention to ventricular cannulation. The scarcity of published reports of clinical complications secondary to cannulation has also attenuated concern for this component of the circuit. However, anecdotal reports of ventricular suction and endocardial trauma suggest that sub-optimal cannulation may be a more common occurrence than reflected in the literature. These studies illustrated the dramatic effect that cannula positioning may have on ventricular suck-down. Further, it was illustrated that the geometric shape of the cannula tip may dramatically affect positional sensitivity to obstruction, and in particular, that a flanged cannula may mitigate this effect. Avoidance of suction would in turn lessen the risk of myocardial trauma and broaden the hemodynamic range in which ventricular decompression can be provided. These preliminary observations motivate consideration of a longitudinal clinical study to corroborate or refute prevailing skepticism about the importance of ventricular cannulation on morbidity and mortality.

\section{Supplementary Material}

Refer to Web version on PubMed Central for supplementary material.

\section{Acknowledgments}

The authors would like to thank Arielle Drummond, PhD; and Fangjun Shu, PhD; for their assistance with the development of the isolated heart preparation. The Authors would also like to thank Harvey Borovetz, PhD for his support. Financial support was provided by an STTR Grant from the National Institute of Health 1R41HL082251-01.

\section{References}

1. Badiwala MV, Ross HJ, Rao V. An unusual complication of support with a continuous-flow cardiac assist device. N Engl J Med. 2007; 357(9):936-7. [PubMed: 17761599]

2. Amin DV, et al. Induction of ventricular collapse by an axial flow blood pump. ASAIO J. 1998; 44(5):M685-90. [PubMed: 9804522]

3. Reesink K, et al. Suction due to left ventricular assist: implications for device control and management. Artif Organs. 2007; 31(7):542-9. [PubMed: 17584479]

4. Miyake Y, et al. Left ventricular mobile thrombus associated with ventricular assist device: diagnosis by transesophageal echocardiography. Circ J. 2004; 68(4):383-4. [PubMed: 15056839]

5. Votapka TV, et al. Left ventricular cannula obstruction in a patient with previous ventricular aneurysmectomy. Ann Thorac Surg. 1994; 58(4):1182-4. [PubMed: 7944781]

6. Griffith BP, et al. HeartMate II left ventricular assist system: from concept to first clinical use. Ann Thorac Surg. 2001; 71(3 Suppl):S116-20. discussion S114-6. [PubMed: 11265845]

7. Ferreira A, et al. A discriminant-analysis-based suction detection system for rotary blood pumps. Conf Proc IEEE Eng Med Biol Soc. 2006; 1:5382-5. [PubMed: 17946698] 
8. Choi S, Boston JR, Antaki JF. Hemodynamic controller for left ventricular assist device based on pulsatility ratio. Artif Organs. 2007; 31(2):114-25. [PubMed: 17298400]

9. Vollkron M, et al. Development of a suction detection system for axial blood pumps. Artif Organs. 2004; 28(8):709-16. [PubMed: 15270952]

10. Watanabe K, et al. Development of a flexible inflow cannula with titanium inflow tip for the NEDO biventricular assist device. ASAIO J. 2004; 50(4):381-6. [PubMed: 15307553]

11. Antaki JF, et al. An improved left ventricular cannula for chronic dynamic blood pump support. Artif Organs. 1995; 19(7):671-5. [PubMed: 8572971]

12. Curtis AS, et al. Novel ventricular apical cannula: in vitro evaluation using transparent, compliant ventricular casts. ASAIO J. 1998; 44(5):M691-5. [PubMed: 9804523]

13. Bhama JK, et al. Development of an ex vivo ovine ventricular assist device model for intraventricular visualization of the inflow cannula. J Heart Lung Transplant. 2009; 28(8):860-1. [PubMed: 19632587]

14. Bachman, T. Biomedical Engineering. University of Pittsburgh; Pittsburgh: 2003. Development and Evaluation of the Quintessential Ventricular Cannula; p. 57

15. Drummond, A. Biomedical Engineering. Carnegie Mellon University; Pittsburgh: 2008. Biomedical surgical planning for pediatric ventricular assist device (PVAD); p. 189

16. Curtis, A. Bioengineering. University of Pittsburgh; Pittsburgh: 1998. A Multiple Modality Study of The Hemodynamics of Left Ventricular Apical Cannulations. 

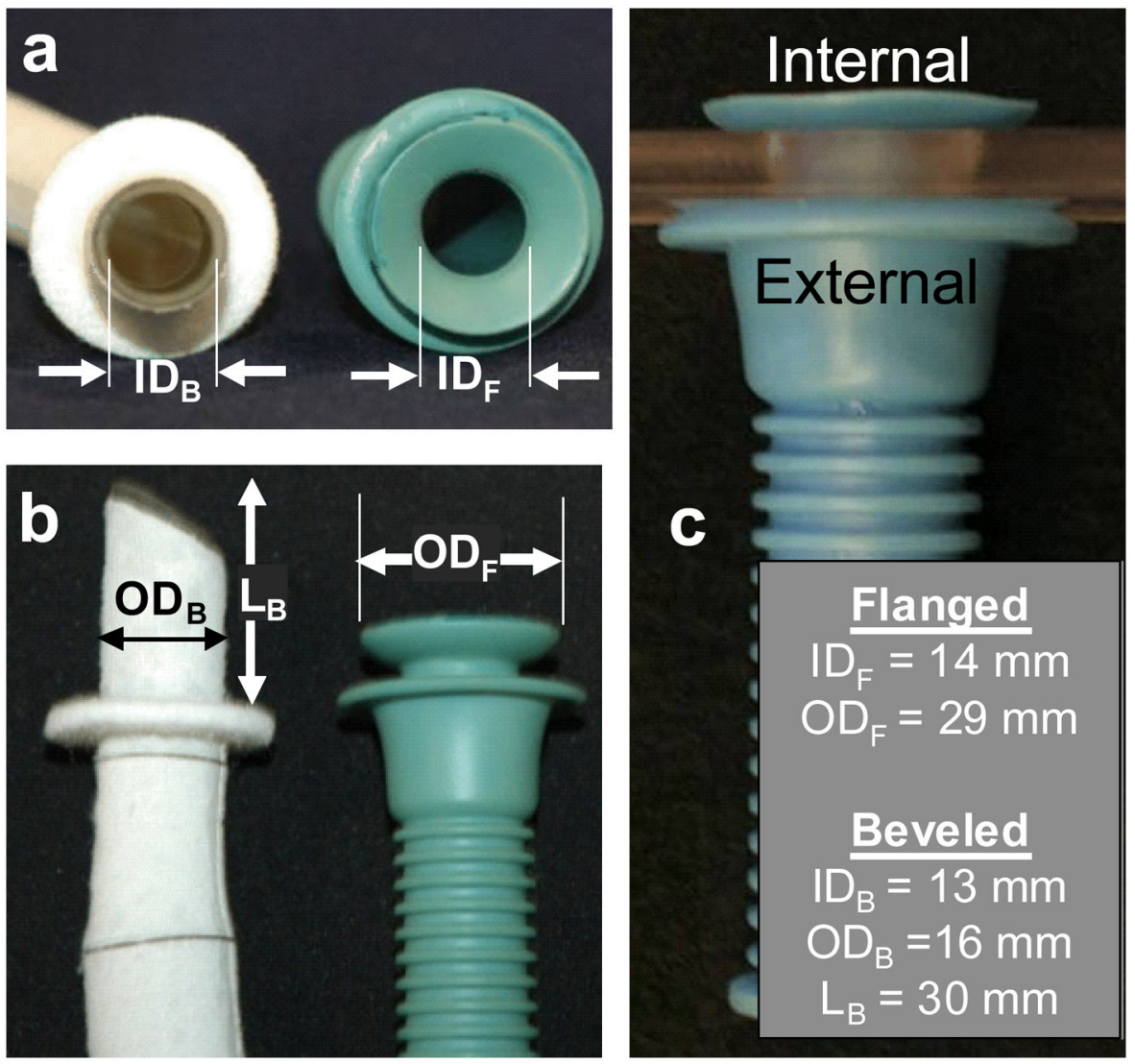

Figure 1.

Conventional beveled ventricular cannula (white) and prototype (blue). (a) axial view; (b) side view; (c) flanged cannula deployed through small diameter opening in Plexiglas, illustrating retention by external flange. 

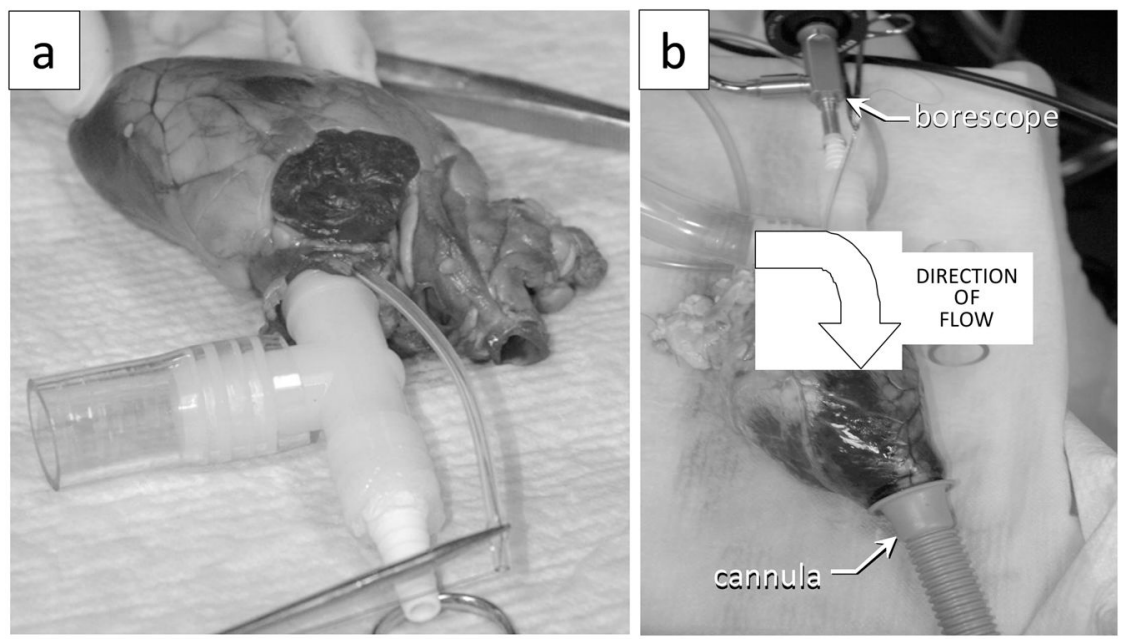

Figure 2.

Ovine Heart Preparation. (a) Tee-fitting attached to atrial remnant and tied with purse string. (b) Circulatory loop with cannulated LV and borescope inserted through T-tube. (Arrow indicates direction of flow.) 

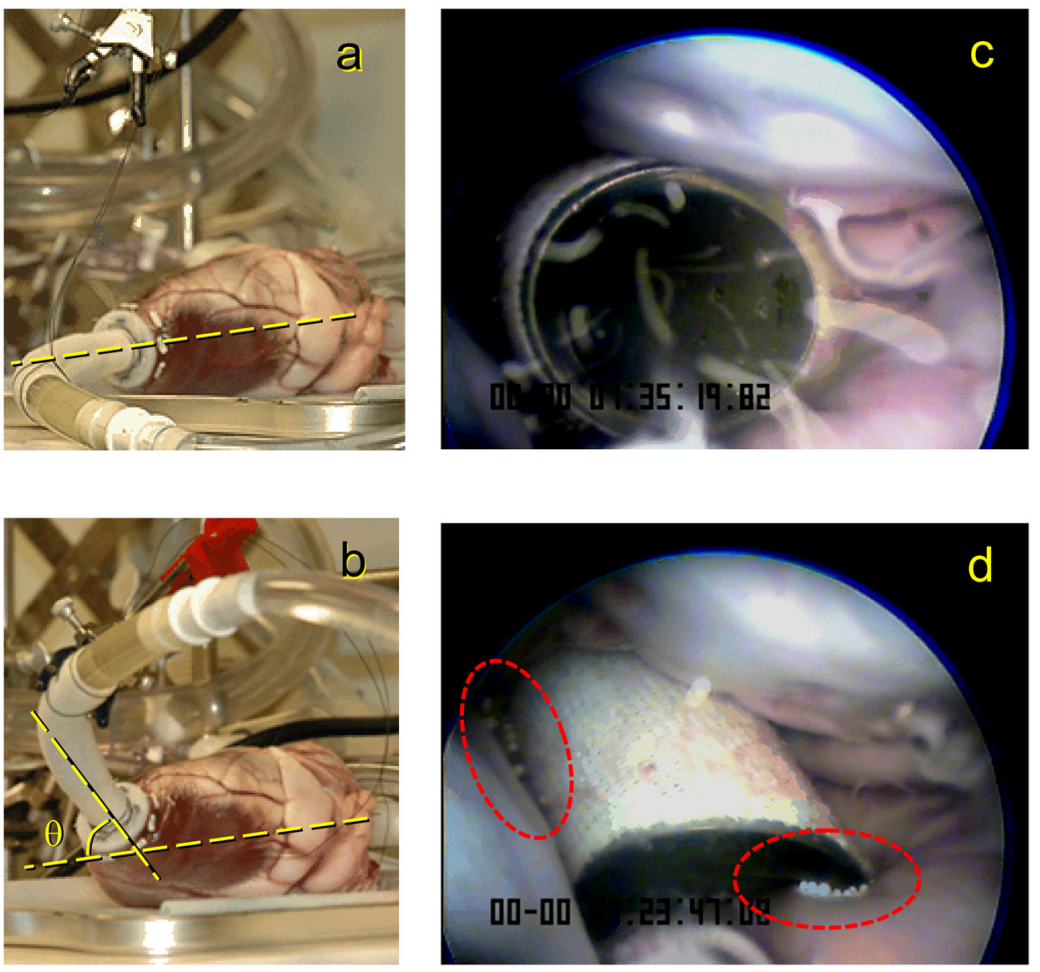

Figure 3.

External view of properly aligned beveled cannula with long axis of ventricle (a) and deflected ventrally by approximately 45 degrees (b). Panels (c) and (d) provide still images of respective particle flow fields generated for the beveled cannula, revealing particle deposition (circled) in the crevice between the cannula and endocardium. 

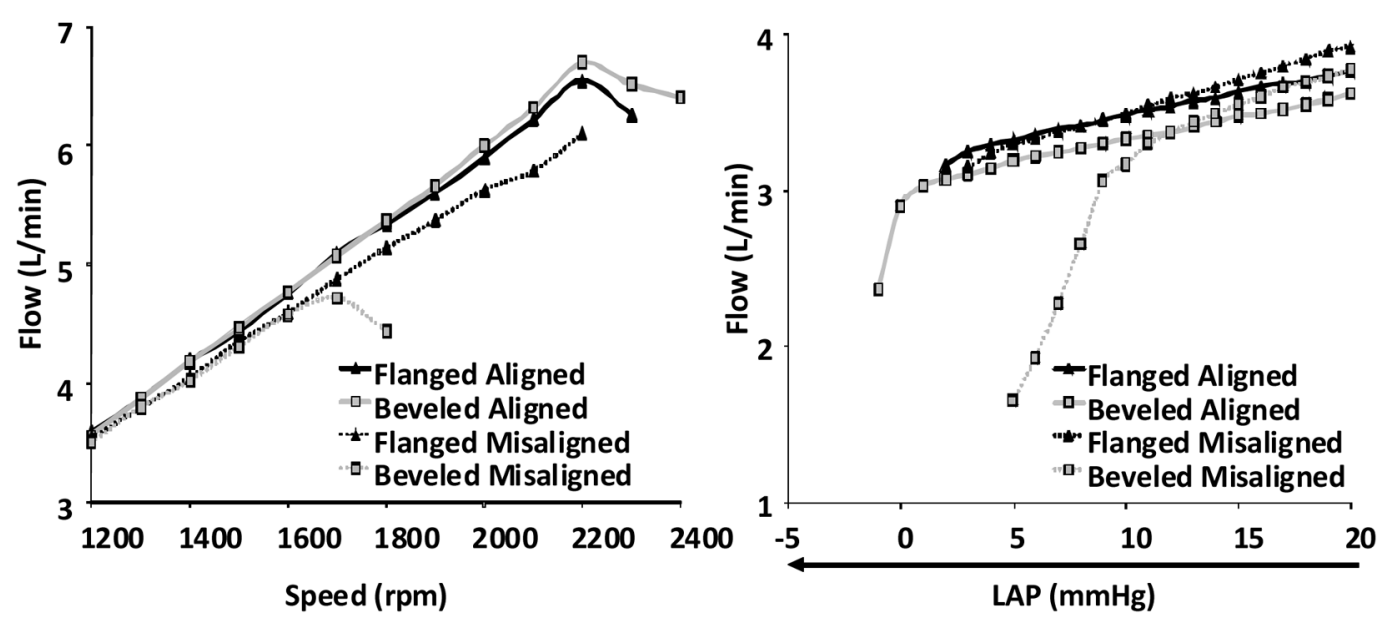

Figure 4.

(Left) Flow versus speed for aligned and misaligned cannulae for initial LAP of 20mmHg up to the point of suction for the second study. (Right) Flow versus Pressure as preload is reduced with pump speed fixed at 1250 RPM for the second study. Arrow indicates time progression for hypovolemic protocol 

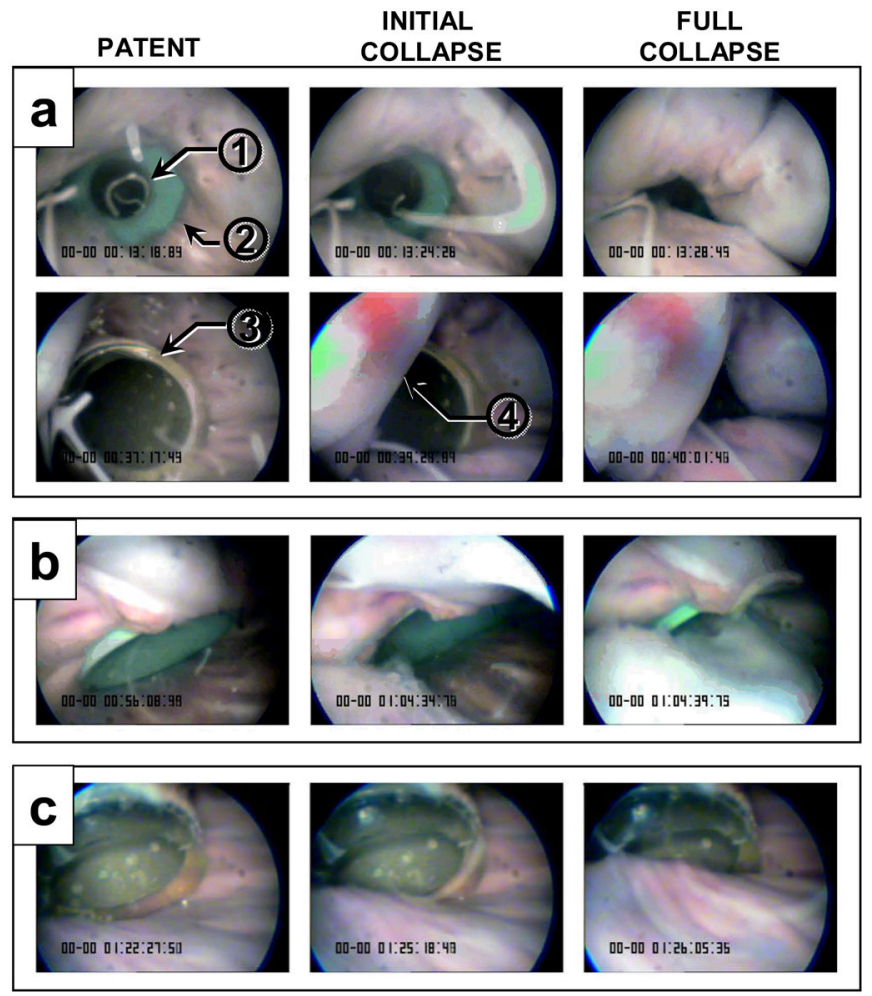

Figure 5.

Still images captured from borescopic video illustrating the progression of the three modes of suction from patent flow path (left) to nearly full collapse (right). (a) Concentric collapse of properly aligned flanged and beveled cannulae, (b) Instantaneous entrainment of a misaligned flanged cannula and (c) Gradual entrainment of a misaligned beveled cannula. View of the cannula lumen (1), flange (2), and bevel (3). Ventricular free wall indicated by arrow (4). 

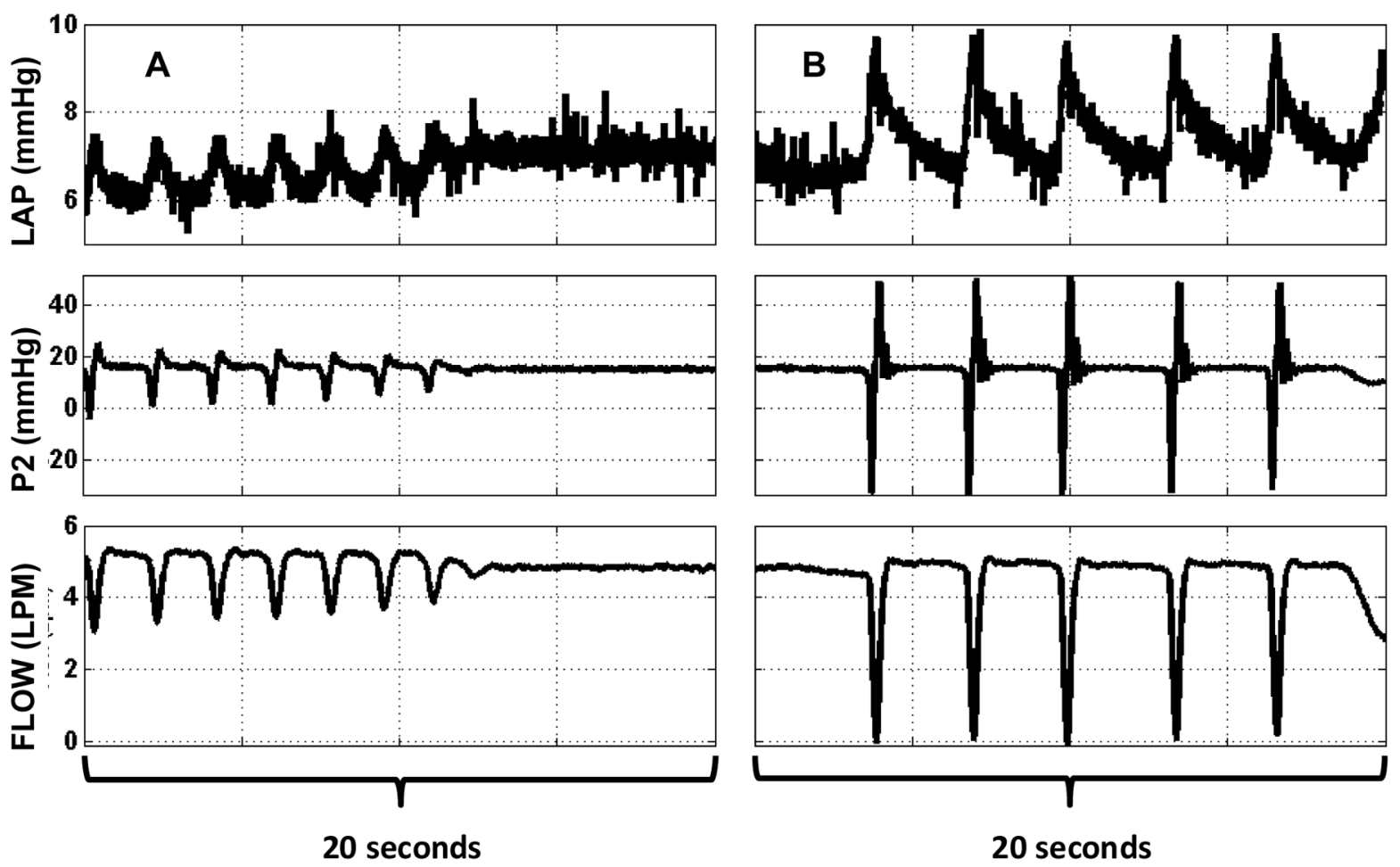

Figure 6.

Examples of hemodynamic instability observed in study \#2. Left column: Dampened, incomplete occlusion. Right column: Total intermittent occlusion. 

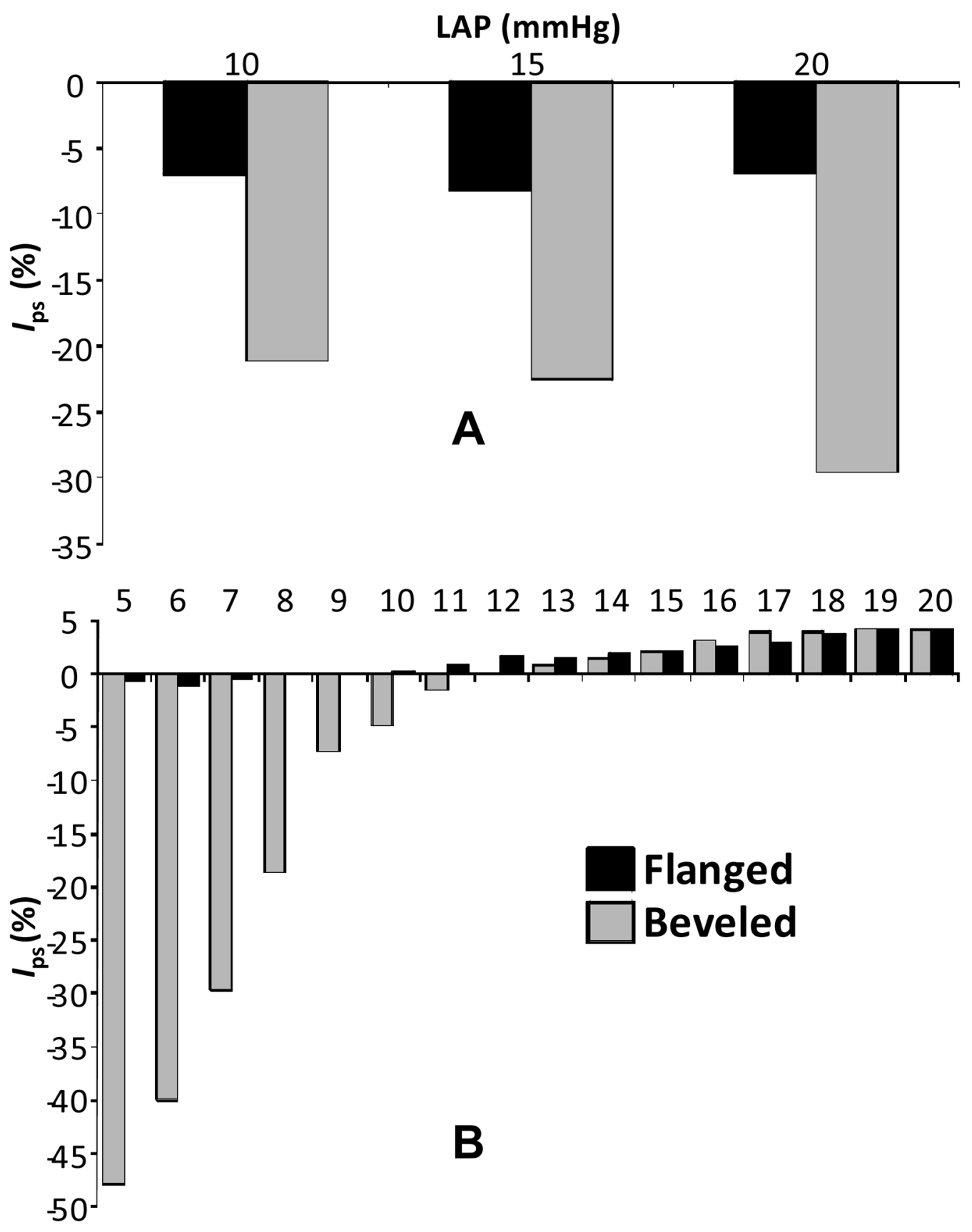

Figure 7.

(Left) Positional sensitivity index Ips for maximal attainable flow (regardless of speed) measured during the over-pumping protocol of the second study. (Right) Ips in flow for the hypovolemic protocol of the second study at a fixed speed of 1250 RPM. Arrow indicates time progression for hypovolemic protocol. 
